\title{
Early Growth Performance Comparison of Weaned and Suckling Murrah Buffalo Calves under Institutional Situations
}

\author{
K.J. Kantharaja, A.K.S. Tomar, O.R. Nataraju* and B.T. Naveen Kumar \\ Division of Livestock Production Management, Indian Veterinary Research Institute, \\ Izatnagar, Bareilly - 243 122, India \\ *Corresponding author
}

\begin{abstract}
A B S T R A C T
Thirteen newly born Murrah buffalo calves were utilized for the experiment and were randomly allocated to suckling and weaned groups. The allocation resulted in 7 suckling calves as well as 6 calves under weaning system of management. The overall least squares'

\begin{tabular}{|c|}
\hline Keywords \\
\hline $\begin{array}{l}\text { Heart girth, Live body } \\
\text { weights, Murrah buffalo } \\
\text { calves, Suckling, } \\
\text { Weaning }\end{array}$ \\
\hline Article Info \\
\hline $\begin{array}{l}\text { Accepted: } \\
\text { 07 April } 2018 \\
\text { Available Online: } \\
\text { 10 May } 2018\end{array}$ \\
\hline
\end{tabular}
means (LSM's) for LWB's of Murrah buffalo calves at birth, 1, 2, 3, 4, 5, 6, 7, 8, 9, 10, 11, $12,13,14,15,16,17,18,19,20,21,22,23,24,25,26,27,28,29,30$ and 31 weeks of age were $30.87 \pm 1.13,37.81 \pm 2.37,41.50 \pm 2.23,44.02 \pm 2.29,46.65$. $\pm 2.35,49.31 \pm 2.57$, $52.54 \pm 2.85,54.46 \pm 2.82,58.65 \pm 3.10,63.56 \pm 3.02,67.73 \pm 3.19,71.35 \pm 3.46,76.53 \pm 3.53$, $79.16 \pm 3.31,84.04 \pm 3.75,89.59 \pm 3.89,93.49 \pm 3.83,99.25 \pm 4.42,103.20 \pm 4.48,108.22 \pm 5.29$, $\begin{array}{llll}113.45 \pm 5.34, & 118.12 \pm 5.25, \quad 123.41 \pm 4.89, & 127.53 \pm 4.68, \quad 127.20 \pm 4.69, & 134.41 \pm 5.37,\end{array}$ $145.18 \pm 3.84,149.59 \pm 3.85,152.95 \pm 3.73,164.42 \pm 5.46$ and $168.48 \pm 5.46 \mathrm{~kg}$, respectively. The effect of rearing system was significant $(\mathrm{P} \leq 0.05)$ on LWT's at 2, 13-15, 24, 27 and 28 weeks of age whereas it was highly significant $(\mathrm{P} \leq 0.01)$ on LWT's at $16-23$ weeks of age. The birth weights were almost same in both the systems of rearing and these were $30.87 \pm 1.09$ and $30.92 \pm 1.17 \mathrm{~kg}$, respectively, for suckling and weaned calves. The significant increase in body weights was initially noticed in suckling system calves at WT2 $(45.58 \pm 2.37 \mathrm{~kg})$ over their weaned counterparts $(38.11 \pm 2.12 \mathrm{~kg})$. The effects of sex and sex (within the rearing system) on weekly live body weights were non-significant.
\end{abstract}

\section{Introduction}

Asian water buffalo (Bubalus bubalis), a large animal with profound genetic variability, plays an important role in the rural economy of India by significantly contributing towards milk, meat, hides and skins along with draught power. India shares $53.6 \%$ of the world buffalo population (FAO, 2009) contributing $56 \%$ of total milk production of the country (FAO, 2008). There are two well-known methods of calf rearing in buffaloes i.e. suckling and weaning. Usually buffalo calves are reared under suckling system wherein assessment of actual milk suckled for optimum growth becomes very difficult. In former system of rearing, calf is allowed to suckle one or two teats before and/or after milking the dam on the other hand in later system, calf is taken away from its mother just immediately after birth or after 2-3 days of birth. In weaning system, the relationship between the dam and young animal is reported to be strengthened as exhibited by increase in 
non-agonistic encounters and the synchronization of their activity (Veissier and Le Neindre, 1989). As a matter of fact, calves which have been weaned for less than two weeks are faster in changing a learned route in a maze compared to those which have been weaned for longer (Veissier et al., 1989a). Generally, in artificial rearing, the calves are weaned as soon as possible mainly for economic reasons e.g. solid food costs less than milk and the provision of solid food is less labour intensive than the provision of milk (Garnsworthy, 2005).

Growth rate is an important trait that determines the age at which an animal will reach puberty and conceive which in turn directly influences the age at first calving which ultimately determines the lifetime productivity of an animal (Lawrence and Fowler, 1997).

Providing calf starter along with restricted milk and skimmed milk is the most effective feeding system. It did not hamper the growth rate of calves as compared to a situation where milk feeding is done for longer periods (Razdan et al., 1965; Wooden et al., 1968; Pachauri et al., 1976; Krishna Mohan et al., 1987 and Saikia et al., 1988).

Weaning studies on beef cattle (Green and Buric, 1953; Fluharty et al., 2000) indicated that early weaned calves had more efficient gains in their body weights than traditionally weaned calves depicting their better carcass conformation (Barker-Neef et al., 2001; Myers et al., 1999b and Wertz et al., 2002).

Similarly, the early weaning resulted in acceptable body weight gains and no adverse health effects in dairy calves (Hopkins, 1997). In addition, early weaning program reduces feed cost because of early transition to dry feeds which are more economical than milk (Yanar and Aydin, 2000). Early weaning might be a feasible alternative for a more costeffective use of feed resources (Peterson et al., 1987). Though, enormous studies on weaning of calves were reported to be beneficial in dairy industry, the information on the effect of weaning on growth performance of Murrah buffalo calves are very scanty and hence the present study was undertaken at Livestock Production and Management Section, Indian Veterinary Research Institute (IVRI), Izatnagar (Uttar Pradesh) to study the effect of weaning on early growth of Murrah buffalo calves in comparison to calves kept under age old suckling system of management.

\section{Materials and Methods}

\section{Source and description of experimental calves}

The relevant data was generated and recoded based on trial made on Murrah buffalo calves reared at Calf Unit of Cattle and Buffalo Farm of Livestock Production and Management Section, Indian Veterinary Research Institute (IVRI), Izatnagar, Bareilly, Uttar Pradesh (India) which is situated in India at $28.22^{\circ} \mathrm{N}$ latitude, $79.22^{\circ} \mathrm{E}$ longitude and at an altitude of 568 feet above mean sea level (MSL). The climatic condition is extremely hot during summers (May-June) and very cold during winters (December-February). The total annual rainfall ranges from 90 to $120 \mathrm{~cm}$. The institute has 165 hectares of well irrigated fodder cultivation land to ensure round the year supply of green fodder to these animals.

As per the plan, all new births in Murrah buffaloes were to be included in suckling/weaned groups, at random. Thirteen newly born Murrah buffalo calves were utilized for the experiment. Those calves were divided in to two experimental groups in such a way so that 7 calves were kept in suckling group and 6 were shifted to weaning system of management. 


\section{Housing management and weaning procedure}

The calves were housed individually in well ventilated, clean and dry pacca pens for 18 hours daily throughout the experimental period. They were let loose for 6 hours daily from $9 A M$ to $3 P M$ in a kachha paddock for exercise and also to facilitate washing, cleaning and drying of shed. There, they had free access to fresh, clean water and calf starter and green fodder. Proper health coverage and preventive measures were followed throughout the experimental period.

Calves were protected against contagious diseases by scheduled vaccination and were treated against internal and external parasites regularly as per the farm practices. Day old weaning was followed for weaning and this group was kept under artificial nursing. The animals which were in suckling group were allowed to suckle their dams all along the lactation period along with provision for calf starter and ad libitum itum green fodder.

\section{Feeding of experimental calves}

\section{Suckling calves}

Each calf was fed with colostrum of its own dam within few hours after birth and twice daily for three days at the rate of $10 \%$ of live body weight and later that was allowed to suckle its dam twice daily during the milking hours. To calculate the total milk consumed by the calf, body weight immediately before and after suckling was taken using electronic weighing balance kept at Calf Unit. Calves were allowed to suckle their dams till the dam was dried up. Along with milk feeding, calves were provided free access to calf starter during morning and night hours. Succulent green fodder and roughages were also made available to these calves as early as possible after initial learning on ad libitum basis.

\section{Weaned calves}

Each calf was fed with colostrum of its own dam within few hours after birth and twice daily for three days at the rate of $10 \%$ of live body weight. Later, it was kept under hand feeding followed by pail feeding till 71 days of age. The feeding schedule for weaned calves is depicted in Table 2.

\section{Classification and statistical analysis of the data}

Weekly live body weights (kg.) of calves were recorded for both the groups (suckling and weaned) using electronic weighing balance. The available data were classified as per the rearing systems (suckling and weaned), sex of calf (males and females) and sex within the rearing system (suckling male, suckling female, weaned male and weaned female). The standard statistical analytical procedures were adopted for analysis of the generated data under study using SAS 9.2 version. The factors considered were system of rearing (suckling and weaned), sex of calf (male or female), and sex within the system of rearing (suckling male/ suckling female and weaned male/weaned female).

\section{Results and Discussion}

Overall weekly live body weights (LBW's) of murrah buffalo calves

The overall least squares' means (LSM's) for LWB's of Murrah buffalo calves at birth (BWT), 1 (WT1), 2(WT2), 3 (WT3), 4 (WT4), 5 (WT5), 6 (WT6), 7 (WT7), 8 (WT8), 9 (WT9), 10 (WT10), 11 (WT11), 12 (WT12), 13 (WT13), 14 (WT14), 15 (WT15), 16 (WT16), 17 (WT17), 18 (WT18), 19 (WT19), 20 (WT20), 21 (WT21), 22 (WT22), 23 (WT23), 24 (WT24), 25 (WT25), 26 (WT26), 27 (WT27), 28 (WT28), 29 (WT29), 30 (WT30) and 31 (WT31) weeks of age were 
$30.87 \pm 1.13, \quad 37.81 \pm \quad 2.37, \quad 41.50 \pm 2.23$, $44.02 \pm 2.29, \quad 46.65 . \quad \pm 2.35, \quad 49.31 \pm 2.57$, $52.54 \pm 2.85, \quad 54.46 \pm 2.82, \quad 58.65 \pm 3.10$, $63.56 \pm 3.02, \quad 67.73 \pm 3.19, \quad 71.35 \pm 3.46$, $76.53 \pm 3.53, \quad 79.16 \pm 3.31, \quad 84.04 \pm 3.75$, $89.59 \pm 3.89, \quad 93.49 \pm 3.83, \quad 99.25 \pm 4.42$, $103.20 \pm 4.48, \quad 108.22 \pm 5.29, \quad 113.45 \pm 5.34$, $118.12 \pm 5.25, \quad 123.41 \pm 4.89, \quad 127.53 \pm 4.68$, $127.20 \pm 4.69, \quad 134.41 \pm 5.37, \quad 145.18 \pm 3.84$, $149.59 \pm 3.85,152.95 \pm 3.73,164.42 \pm 5.46$ and $168.48 \pm 5.46 \mathrm{~kg}$, respectively (Table 1 ).

\section{Effect of system of rearing on weekly live body weights in Murrah buffalo calves}

The effect of rearing system was found to be significant $(\mathrm{P} \leq 0.05)$ on WT2, WT13, WT14, WT15, WT24, WT27 and WT28 whereas it was highly significant $(\mathrm{P} \leq 0.01)$ on LWT's at WT16, WT17, WT18, WT19, WT20, WT 21, WT22 and WT23 (Table 1 and Fig. 1). The birth weights were almost same in both the systems of rearing and these were $30.87 \pm 1.09$ and $30.92 \pm 1.17 \mathrm{~kg}$, respectively, for suckling and weaned calves (Table 1). The significant increase in body weights was initially noticed in suckling system calves at WT2 $(45.58 \pm 2.37$ $\mathrm{kg}$ ) over their weaned counterparts $(38.11 \pm 2.12 \mathrm{~kg}$, Table 1).

There were almost same birth weights in calves of both the systems of rearing but significant increase in body weights was noticed in suckling calves at WT2 and later it increased gradually up to WT13. This point onwards it increased significantly compared to their weaned counterparts and highly significant effect $(\mathrm{P} \leq 0.01)$ was noticed at WT16, WT17, WT18, WT19, WT20, WT21 and WT22. There was a gap of almost $28 \mathrm{~kg}$ between calves of two rearing systems at WT26 and the suckling calves were superior to weaned calves. The weaned calves expressed gradual improvement in the BW's during the experimental weeks but it was lesser than their suckling counterparts (Table 2 and Fig. 1). The superior LBW's expressed by suckling system calves may be due the fact that the calves under suckling system had been offered full milk of buffalo under suckling process which consists of higher fat and total solids percentages whereas in weaned system of management calves were fed mixed milk of cow and buffaloes where fat and total solid percentages are comparatively lower. At the same time, it may also be due to longer duration of milk feeding in suckling system of management as compared to that of weaning system of management where the calves were fed milk only for 71 days. It must be stressed upon that the most important factor which might affect the growth of calves and especially for weaned ones at early stages is the intensive care and management of these calves during this period. Similar to the present findings, Khoury et al., (1967) in buffalo calves reported that LBW's of early weaned calves were inferior to those of late weaned calves. However, present results were not in agreement with the observations made by the Myers et al., (1999) and Wertz et al., (2002), who reported that early weaned calves had more gains than traditionally weaned calves. Calves that were heavier at birth were significantly heavier at 4 and 8 months of age and the gain up to 8 months of age was not significantly influenced by birth weight differences. Canadian Holstein calves left with their dams for $14 \mathrm{~d}$ after birth were almost 29 $\mathrm{lb}$ heavier at $14 \mathrm{~d}$ of age than calves removed from the dam on day 1 and who were fed limited whole milk at the rate of $10 \%$ of LBW on daily basis (Flower and Weary, 2001).Studies with twice-daily hand feeding of milk replacer also showed that ad libitum intake of milk was in excess (18\% of LBW). Khouri and Pickering (1968) fed whole milk to calves during the first 6 weeks of life at the rate of 11.3, 13.9, 15.9 and/or 19.4\% (ad libitum) of LBW. Daily LBW gains during weeks 2 to 6 of life were $0.90,1.10,1.37$ and $2.07 \mathrm{lb} / \mathrm{d}$ respectively. 
Table.1 Least squares means (LSM) for weekly live body weight of Murrah buffalo calve

\begin{tabular}{|c|c|c|c|c|c|c|c|c|c|c|c|}
\hline & & & $\mathrm{LSM} \pm \mathrm{SE}(\mathbf{k g})$ & & & & & & & & \\
\hline Particulars & BWT & WT1 & WT2 & WT3 & WT4 & WT5 & WT6 & WT7 & WT8 & WT9 & WT10 \\
\hline $\begin{array}{l}\text { Overall } \\
\text { mean }(\mu)\end{array}$ & $\begin{array}{c}30.87 \pm 1.13 \\
(13)\end{array}$ & $\begin{array}{c}37.81 \pm 2.37 \\
(13)\end{array}$ & $\begin{array}{l}41.50 \pm 2.23 \\
(11)\end{array}$ & $\begin{array}{c}44.02 \pm 2.29 \\
\text { (11) }\end{array}$ & $\begin{array}{l}46.65 \pm 2.35 \\
\text { (11) }\end{array}$ & $\begin{array}{c}49.31 \pm 2.57 \\
(11)\end{array}$ & $\begin{array}{c}52.54 \pm 2.85 \\
(11)\end{array}$ & $\begin{array}{c}55.46 \pm 2.82 \\
(11)\end{array}$ & $\begin{array}{c}58.65 \pm 3.10 \\
(11)\end{array}$ & $\begin{array}{c}63.56 \pm 3.02 \\
(11)\end{array}$ & $\begin{array}{l}67.73 \pm 3.19 \\
\quad(11)\end{array}$ \\
\hline \multicolumn{12}{|c|}{ Systems of rearing } \\
\hline & NS & NS & $*$ & NS & NS & NS & NS & NS & NS & NS & NS \\
\hline $\begin{array}{l}\text { Suckling } \\
\text { system }\end{array}$ & $\begin{array}{l}30.83 \pm 1.09 \\
\text { (7) }\end{array}$ & $\begin{array}{l}39.72 \pm 2.30 \\
\quad(7)\end{array}$ & $\begin{array}{l}45.58 \pm 2.37 \\
(5)\end{array}$ & $\begin{array}{l}47.72 \pm 2.43 \\
\quad(5)\end{array}$ & $\begin{array}{l}49.96 \pm 2.50 \\
(5)\end{array}$ & $\begin{array}{l}52.10 \pm 2.73 \\
(5)\end{array}$ & $\begin{array}{c}55.63 \pm 3.03 \\
(5)\end{array}$ & $\begin{array}{c}58.51 \pm 3.00 \\
(5)\end{array}$ & $\begin{array}{c}62.92 \pm 3.29 \\
(5)\end{array}$ & $\begin{array}{c}68.69 \pm 3.21 \\
(5)\end{array}$ & $\begin{array}{c}73.73 \pm 3.38 \\
\text { (5) }\end{array}$ \\
\hline $\begin{array}{l}\text { Weaning } \\
\text { system }\end{array}$ & $\begin{array}{l}30.92 \pm 1.17 \\
(6)\end{array}$ & $\begin{array}{l}35.59 \pm 2.46 \\
(6)\end{array}$ & $\begin{array}{c}38.11 \pm 2.12 \\
(6)\end{array}$ & $\begin{array}{l}40.94 \pm 2.17 \\
(6)\end{array}$ & $\begin{array}{c}43.89 \pm 2.23 \\
(6)\end{array}$ & $\begin{array}{l}46.99 \pm 2.44 \\
(6)\end{array}$ & $\begin{array}{c}49.97 \pm 2.70 \\
(6)\end{array}$ & $\begin{array}{c}52.91 \pm 2.68 \\
(6)\end{array}$ & $\begin{array}{c}55.09 \pm 2.94 \\
(6)\end{array}$ & $\begin{array}{c}59.28 \pm 2.87 \\
(6)\end{array}$ & $\begin{array}{c}62.73 \pm 3.03 \\
(6)\end{array}$ \\
\hline \multicolumn{12}{|l|}{ Sex of calf } \\
\hline & NS & NS & NS & NS & NS & NS & NS & NS & NS & NS & NS \\
\hline Males & $\begin{array}{c}31.83 \pm 1.09 \\
(7)\end{array}$ & $\begin{array}{l}37.71 \pm 2.30 \\
\quad(7)\end{array}$ & $\begin{array}{c}41.79 \pm 2.12 \\
(6)\end{array}$ & $\begin{array}{l}43.28 \pm 2.17 \\
(6)\end{array}$ & $\begin{array}{c}45.30 \pm 2.23 \\
(6)\end{array}$ & $\begin{array}{l}48.07 \pm 2.44 \\
\quad(6)\end{array}$ & $\begin{array}{c}51.41 \pm 2.70 \\
(6)\end{array}$ & $\begin{array}{c}54.41 \pm 2.68 \\
(6)\end{array}$ & $\begin{array}{c}57.39 \pm 2.94 \\
(6)\end{array}$ & $\begin{array}{c}61.89 \pm 2.8 \\
(6)\end{array}$ & $\begin{array}{c}65.36 \pm 3.03 \\
(6)\end{array}$ \\
\hline Females & $29.92 \pm 1.17(6)$ & $\begin{array}{c}37.60 \pm 2.46 \\
(6)\end{array}$ & $\begin{array}{l}41.90 \pm 2.37 \\
(5)\end{array}$ & $\begin{array}{l}45.38 \pm 2.43 \\
(5)\end{array}$ & $\begin{array}{c}48.55 \pm 2.50 \\
(5)\end{array}$ & $\begin{array}{l}51.02 \pm 2.73 \\
(5)\end{array}$ & $\begin{array}{c}54.20 \pm 3.03 \\
(5)\end{array}$ & $\begin{array}{c}57.01 \pm 3.00 \\
(5)\end{array}$ & $\begin{array}{c}60.62 \pm 3.29 \\
(5)\end{array}$ & $\begin{array}{c}66.09 \pm 3.21 \\
(5)\end{array}$ & $\begin{array}{c}71.10 \pm 3.38 \\
(5)\end{array}$ \\
\hline \multicolumn{12}{|c|}{ Sex within the system of rearing } \\
\hline & NS & NS & NS & NS & NS & NS & NS & NS & NS & NS & NS \\
\hline $\begin{array}{l}\text { Suckling } \\
\text { Males }\end{array}$ & $\begin{array}{l}32.00 \pm 1.43 \\
(4)\end{array}$ & $\begin{array}{l}38.62 \pm 3.00 \\
\text { (4) }\end{array}$ & $\begin{array}{c}44.83 \pm 2.99 \\
\text { (3) }\end{array}$ & $\begin{array}{l}45.77 \pm 3.07 \\
\text { (3) }\end{array}$ & $\begin{array}{c}47.00 \pm 3.16 \\
\text { (3) }\end{array}$ & $\begin{array}{l}48.98 \pm 3.45 \\
\text { (3) }\end{array}$ & $\begin{array}{c}52.47 \pm 3.84 \\
\text { (3) }\end{array}$ & $\begin{array}{c}55.03 \pm 3.80 \\
\text { (3) }\end{array}$ & $\begin{array}{c}59.03 \pm 4.16 \\
\text { (3) }\end{array}$ & $\begin{array}{c}64.82 \pm 4.06 \\
\text { (3) }\end{array}$ & $\begin{array}{c}70.08 \pm 4.28 \\
\text { (3) }\end{array}$ \\
\hline $\begin{array}{l}\text { Suckling } \\
\text { Females }\end{array}$ & $\begin{array}{l}29.67 \pm 1.65 \\
\text { (3) }\end{array}$ & $\begin{array}{l}40.82 \pm 3.47 \\
\text { (3) }\end{array}$ & $\begin{array}{l}46.32 \pm 3.67 \\
\text { (2) }\end{array}$ & $\begin{array}{c}49.67 \pm 3.76 \\
\text { (2) }\end{array}$ & $\begin{array}{l}52.92 \pm 3.87 \\
\text { (2) }\end{array}$ & $\begin{array}{l}55.23 \pm 4.23 \\
\text { (2) }\end{array}$ & $\begin{array}{c}58.80 \pm 4.70 \\
(2)\end{array}$ & $\begin{array}{c}61.98 \pm 4.65 \\
\text { (2) }\end{array}$ & $\begin{array}{c}66.81 \pm 5.10 \\
(2)\end{array}$ & $\begin{array}{c}72.56 \pm 4.97 \\
\text { (2) }\end{array}$ & $\begin{array}{c}77.38 \pm 5.24 \\
\text { (2) }\end{array}$ \\
\hline $\begin{array}{l}\text { Weaned } \\
\text { Males }\end{array}$ & $\begin{array}{c}31.67 \pm 1.45 \\
\text { (3) }\end{array}$ & $\begin{array}{c}36.80 \pm 3.47 \\
\text { (3) }\end{array}$ & $\begin{array}{c}38.75 \pm 2.99 \\
\text { (3) }\end{array}$ & $\begin{array}{l}49.67 \pm 3.76 \\
\text { (3) }\end{array}$ & $\begin{array}{c}43.60 \pm 3.16 \\
(3)\end{array}$ & $\begin{array}{l}47.17 \pm 3.45 \\
\text { (3) }\end{array}$ & $\begin{array}{c}50.35 \pm 3.84 \\
\text { (3) }\end{array}$ & $\begin{array}{c}53.78 \pm 3.80 \\
\text { (3) }\end{array}$ & $\begin{array}{c}55.76 \pm 4.16 \\
\text { (3) }\end{array}$ & $\begin{array}{c}58.95 \pm 4.06 \\
\text { (3) }\end{array}$ & $\begin{array}{c}60.64 \pm 4.28 \\
\text { (3) }\end{array}$ \\
\hline $\begin{array}{l}\text { Weaned } \\
\text { Females }\end{array}$ & $\begin{array}{l}30.17 \pm 1.65 \\
\text { (3) }\end{array}$ & $\begin{array}{l}34.38 \pm 3.47 \\
\text { (3) }\end{array}$ & $\begin{array}{l}37.47 \pm 2.99 \\
\text { (3) }\end{array}$ & $\begin{array}{l}41.10 \pm 3.07 \\
\text { (3) }\end{array}$ & $\begin{array}{c}44.17 \pm 3.16 \\
\text { (3) }\end{array}$ & $\begin{array}{l}46.81 \pm 3.45 \\
\text { (3) }\end{array}$ & $\begin{array}{c}49.59 \pm 3.84 \\
\text { (3) }\end{array}$ & $\begin{array}{c}52.05 \pm 3.80 \\
\text { (3) }\end{array}$ & $\begin{array}{c}54.43 \pm 4.16 \\
\text { (3) }\end{array}$ & $\begin{array}{c}59.62 \pm 4.06 \\
\text { (3) }\end{array}$ & $\begin{array}{c}64.83 \pm 4.28 \\
\text { (3) }\end{array}$ \\
\hline
\end{tabular}

*: Significant at $\mathrm{P} \leq 0.05 ; * *$ : Significant at $\mathrm{P} \leq 0.01 ; \mathrm{NS}$ : Non significant 
Continued...

\begin{tabular}{|c|c|c|c|c|c|c|c|c|c|c|}
\hline & $\begin{array}{c}\text { LSM } \pm \\
\text { SE } \\
(\mathbf{k g})\end{array}$ & & & & & & & & & \\
\hline $\begin{array}{l}\text { Particula } \\
\text { rs }\end{array}$ & WT11 & WT12 & WT13 & WT14 & WT15 & WT16 & WT17 & WT18 & WT19 & WT20 \\
\hline $\begin{array}{c}\text { Overall } \\
\text { mean }(\mu)\end{array}$ & $\begin{array}{c}71.35 \pm \\
3.46 \\
(11)\end{array}$ & $\begin{array}{c}74.53 \pm 3.5 \\
3(11)\end{array}$ & $\begin{array}{c}79.16 \pm 3.3 \\
1(11)\end{array}$ & $\begin{array}{c}84.04 \pm 3.7 \\
5(11)\end{array}$ & $\begin{array}{c}89.53 \pm 3.89 \\
(11)\end{array}$ & $\begin{array}{c}93.49 \pm 3.8 \\
3(10)\end{array}$ & $\begin{array}{c}99.25 \pm 4.4 \\
2(9)\end{array}$ & $\begin{array}{c}103.20 \pm 4 \\
48(9)\end{array}$ & $\begin{array}{c}108.22 \pm 5 . \\
29(9)\end{array}$ & $\begin{array}{l}113.45 \pm \\
5.34(9)\end{array}$ \\
\hline \multicolumn{11}{|c|}{ Systems of rearing } \\
\hline & NS & NS & $*$ & $*$ & $*$ & $* *$ & $* *$ & $* *$ & $* *$ & $* *$ \\
\hline $\begin{array}{l}\text { Suckling } \\
\text { system }\end{array}$ & $\begin{array}{c}77.22 \pm 3 \\
.67(5)\end{array}$ & $\begin{array}{c}80.34 \pm 3.75 \\
(5)\end{array}$ & $\begin{array}{c}85.89 \pm 3.51 \\
(5)\end{array}$ & $\begin{array}{c}91.99 \pm 3.98 \\
(5)\end{array}$ & $\begin{array}{c}98.67 \pm 4.13 \\
(5)\end{array}$ & $\begin{array}{c}103.87 \pm 3 \\
83(5)\end{array}$ & $\begin{array}{c}109.60 \pm 4 . \\
24(5)\end{array}$ & $\begin{array}{c}114.10 \pm 4 . \\
30(5)\end{array}$ & $\begin{array}{c}119.91 \pm 5 . \\
08(5)\end{array}$ & $\begin{array}{l}126.12 \pm \\
5.12(5)\end{array}$ \\
\hline $\begin{array}{l}\text { Weanin } \\
\text { g system }\end{array}$ & $\begin{array}{c}66.46 \pm 3 \\
.29(6)\end{array}$ & $\begin{array}{c}69.69 \pm 3.35 \\
(6)\end{array}$ & $\begin{array}{c}73.54 \pm 3.1 \\
4(6)\end{array}$ & $\begin{array}{c}77.42 \pm 3.5 \\
6(6)\end{array}$ & $\begin{array}{c}81.91 \pm 3.70 \\
\text { (6) }\end{array}$ & $\begin{array}{c}83.10 \pm 3.83 \\
(5)\end{array}$ & $\begin{array}{c}6.31 \pm 4.64 \\
(4)\end{array}$ & $\begin{array}{c}89.56 \pm 4.7 \\
1(4)\end{array}$ & $\begin{array}{c}93.60 \pm 5.5 \\
6(4)\end{array}$ & $\begin{array}{c}97.62 \pm 5 \\
.61(4)\end{array}$ \\
\hline \multicolumn{11}{|c|}{ Sex of calf } \\
\hline & NS & NS & NS & NS & NS & NS & NS & NS & NS & NS \\
\hline Males & $\begin{array}{c}68.68 \pm 3 \\
.29(6)\end{array}$ & $\begin{array}{c}72.44 \pm 3.35 \\
\text { (6) }\end{array}$ & $\begin{array}{c}76.92 \pm 3.1 \\
4(6)\end{array}$ & $\begin{array}{c}80.24 \pm 3.5 \\
6(6)\end{array}$ & $\begin{array}{c}86.09 \pm 3.70 \\
(6)\end{array}$ & $\begin{array}{c}87.69 \pm 3.8 \\
3(5)\end{array}$ & $\begin{array}{c}.85 \pm 4.24 \\
(5)\end{array}$ & $\begin{array}{c}96.94 \pm 4.3 \\
0(5)\end{array}$ & $\begin{array}{c}101.09 \pm 5 . \\
08(5)\end{array}$ & $\begin{array}{l}107.09 \pm \\
5.12(5)\end{array}$ \\
\hline Females & $\begin{array}{c}75.00 \pm 3 \\
.68(5)\end{array}$ & $\begin{array}{c}77.59 \pm 3.75 \\
(5)\end{array}$ & $\begin{array}{c}82.51 \pm 3.51 \\
(5)\end{array}$ & $\begin{array}{c}89.16 \pm 3.98 \\
(5)\end{array}$ & $\begin{array}{c}94.50 \pm 4.13 \\
(5)\end{array}$ & $\begin{array}{c}99.28 \pm 3.8 \\
3(5)\end{array}$ & $\begin{array}{c}103.06 \pm 4 . \\
64(4)\end{array}$ & $\begin{array}{c}106.72 \pm 4 \\
71(4)\end{array}$ & $\begin{array}{c}112.44 \pm 5 \\
56(4)\end{array}$ & $\begin{array}{l}116.65 \pm \\
5.61(4)\end{array}$ \\
\hline \multicolumn{11}{|c|}{ Sex within the system of rearing } \\
\hline & NS & NS & NS & NS & NS & NS & NS & NS & NS & NS \\
\hline $\begin{array}{c}\text { Suckling } \\
\text { Males }\end{array}$ & $\begin{array}{c}73.44 \pm 4 \\
.65(3)\end{array}$ & $\begin{array}{c}77.04 \pm 4.7 \\
4(3)\end{array}$ & $\begin{array}{c}81.74 \pm 4.4 \\
4(3)\end{array}$ & $\begin{array}{c}90.33 \pm 1.1 \\
6(3)\end{array}$ & $\begin{array}{c}92.09 \pm 5.23 \\
\text { (3) }\end{array}$ & $\begin{array}{c}96.85 \pm 4.84 \\
\text { (3) }\end{array}$ & $\begin{array}{c}103.60 \pm 5 \\
36(3)\end{array}$ & $\begin{array}{c}108.94 \pm 5 \\
44(3)\end{array}$ & $\begin{array}{c}113.83 \pm 6 \\
42(3)\end{array}$ & $\begin{array}{l}121.77 \pm \\
6.48(3)\end{array}$ \\
\hline $\begin{array}{l}\text { Suckling } \\
\text { Females }\end{array}$ & $\begin{array}{c}80.99 \pm 5 \\
.69(2)\end{array}$ & $\begin{array}{c}83.65 \pm 5.8 \\
1(2)\end{array}$ & $\begin{array}{c}90.04 \pm 5.4 \\
4(2)\end{array}$ & $\begin{array}{c}90.50 \pm 1.4 \\
3(2)\end{array}$ & $\begin{array}{c}105.25 \pm 6.4 \\
1(2)\end{array}$ & $\begin{array}{c}110.89 \pm 5 . \\
93(2)\end{array}$ & $\begin{array}{c}115.61 \pm 6 . \\
56(2)\end{array}$ & $\begin{array}{c}119.27 \pm 6 . \\
67(2)\end{array}$ & $\begin{array}{c}125.99 \pm 7 \\
87(2)\end{array}$ & $\begin{array}{l}130.47 \pm \\
7.93(2)\end{array}$ \\
\hline $\begin{array}{l}\text { Weaned } \\
\text { Males }\end{array}$ & $\begin{array}{c}63.92 \pm 4 \\
.65(3)\end{array}$ & $\begin{array}{c}67.85 \pm 4.7 \\
4(3)\end{array}$ & $\begin{array}{c}72.11 \pm 4.4 \\
4(3)\end{array}$ & $\begin{array}{c}86.67 \pm 1.1 \\
6(3)\end{array}$ & $\begin{array}{c}80.09 \pm 5.23 \\
\text { (3) }\end{array}$ & $\begin{array}{c}78.52 \pm 5.93 \\
\text { (2) }\end{array}$ & $\begin{array}{c}82.10 \pm 6.5 \\
6(2)\end{array}$ & $\begin{array}{c}84.95 \pm 6.6 \\
7(2)\end{array}$ & $\begin{array}{c}88.34 \pm 7.8 \\
7(2)\end{array}$ & $\begin{array}{c}92.41 \pm 7 \\
.93(2)\end{array}$ \\
\hline $\begin{array}{l}\text { Weaned } \\
\text { Females }\end{array}$ & $\begin{array}{c}68.99 \pm 4 \\
.65(3)\end{array}$ & $\begin{array}{c}71.52 \pm 4.7 \\
4(3)\end{array}$ & $\begin{array}{c}74.98 \pm 4.4 \\
4(3)\end{array}$ & $\begin{array}{c}87.67 \pm 1.1 \\
6(3)\end{array}$ & $\begin{array}{c}83.74 \pm 5.23 \\
\text { (3) }\end{array}$ & $\begin{array}{c}87.68 \pm 4.84 \\
\text { (3) }\end{array}$ & $\begin{array}{c}90.50 \\
\pm 6.56(2)\end{array}$ & $\begin{array}{c}94.17 \pm 6.6 \\
7(2)\end{array}$ & $\begin{array}{c}98.86 \pm 7.8 \\
7(2)\end{array}$ & $\begin{array}{l}102.83 \pm \\
7.93(2)\end{array}$ \\
\hline
\end{tabular}


Continued...

\begin{tabular}{|c|c|c|c|c|c|c|c|c|c|c|c|}
\hline & $\begin{array}{c}\mathrm{LSM} \pm \mathrm{SE} \\
(\mathrm{kg})\end{array}$ & & & & & & & & & & \\
\hline $\begin{array}{c}\text { Particul } \\
\text { ars } \\
\end{array}$ & WT21 & WT22 & WT23 & WT24 & WT25 & WT26 & WT27 & WT28 & WT29 & WT30 & WT31 \\
\hline $\begin{array}{c}\text { Overall } \\
\text { mean } \\
(\boldsymbol{\mu}) \\
\end{array}$ & $\begin{array}{c}118.12 \pm 5 \\
.25(9)\end{array}$ & $\begin{array}{l}123.41 \pm \\
4.89(8)\end{array}$ & $\begin{array}{l}127.53 \pm \\
4.68(8)\end{array}$ & $\begin{array}{l}127.20 \pm \\
4.69(7)\end{array}$ & $\begin{array}{l}134.41 \pm \\
5.37(6)\end{array}$ & $\begin{array}{c}139.14 \pm 5 \\
.50(6)\end{array}$ & $\begin{array}{l}145.18 \pm \\
3.84(5)\end{array}$ & $\begin{array}{l}149.59 \pm \\
3.85(5)\end{array}$ & $\begin{array}{l}152.95 \pm \\
3.73(5)\end{array}$ & $\begin{array}{l}164.42 \pm \\
5.46(4)\end{array}$ & $\begin{array}{l}168.48 \pm \\
5.46(4)\end{array}$ \\
\hline \multicolumn{12}{|c|}{ Systems of rearing } \\
\hline & $* *$ & $* *$ & $* *$ & $*$ & NS & NS & $*$ & NS & NS & - & - \\
\hline $\begin{array}{c}\text { Sucklin } \\
\text { g } \\
\text { system } \\
\end{array}$ & $\begin{array}{c}131.68 \pm 5 \\
.03(5)\end{array}$ & $\begin{array}{l}137.08 \pm \\
4.34(5)\end{array}$ & $\begin{array}{l}141.48 \pm \\
4.15(5)\end{array}$ & $\begin{array}{l}141.28 \pm \\
4.57(4)\end{array}$ & $\begin{array}{l}144.38 \pm \\
5.00(4)\end{array}$ & $\begin{array}{c}148.59 \pm 5 \\
.12(4)\end{array}$ & $\begin{array}{l}152.90 \pm \\
4.81(4)\end{array}$ & $\begin{array}{l}157.24 \pm \\
4.81 \text { (4) }\end{array}$ & $\begin{array}{l}160.32 \pm \\
4.66(4)\end{array}$ & $\begin{array}{l}164.42 \pm \\
5.46(4)\end{array}$ & $\begin{array}{l}168.48 \pm \\
5.46(4)\end{array}$ \\
\hline $\begin{array}{c}\text { Weanin } \\
\text { g } \\
\text { system }\end{array}$ & $\begin{array}{c}101.16 \pm 5 \\
.52(4)\end{array}$ & $\begin{array}{l}100.64 \pm \\
5.82(3)\end{array}$ & $\begin{array}{l}104.27 \pm \\
5.57(3)\end{array}$ & $\begin{array}{l}108.54 \pm \\
4.85(3)\end{array}$ & $\begin{array}{l}114.46 \pm \\
6.12(2)\end{array}$ & $\begin{array}{c}120.25 \pm 6 \\
.27(2)\end{array}$ & $\begin{array}{c}114.30 \\
(1)\end{array}$ & $\begin{array}{c}119.11 \\
\text { (1) }\end{array}$ & $\begin{array}{c}123.49 \\
\text { (1) }\end{array}$ & - & - \\
\hline \multicolumn{12}{|c|}{ Sex of calf } \\
\hline & NS & NS & NS & NS & NS & NS & NS & NS & NS & NS & NS \\
\hline Males & $\begin{array}{c}112.95 \pm 5 \\
.03(5)\end{array}$ & $\begin{array}{l}113.06 \pm \\
5.47(4)\end{array}$ & $\begin{array}{l}117.50 \pm \\
5.25(4)\end{array}$ & $\begin{array}{l}122.28 \pm \\
4.57(4)\end{array}$ & $\begin{array}{l}125.99 \pm \\
5.00(4)\end{array}$ & $\begin{array}{c}130.92 \pm 5 \\
.12(4)\end{array}$ & $\begin{array}{l}134.89 \pm \\
4.81(4)\end{array}$ & $\begin{array}{l}139.57 \pm \\
4.81(4)\end{array}$ & $\begin{array}{l}143.24 \pm \\
4.66(4)\end{array}$ & $\begin{array}{l}167.61 \pm \\
5.46(3)\end{array}$ & $\begin{array}{l}172.18 \pm \\
5.46(3)\end{array}$ \\
\hline Females & $\begin{array}{c}119.90 \pm 5 \\
.52(4)\end{array}$ & $\begin{array}{l}124.67 \pm \\
4.75(4)\end{array}$ & $\begin{array}{l}128.26 \pm \\
4.55(4)\end{array}$ & $\begin{array}{l}127.54 \pm \\
4.85(3)\end{array}$ & $\begin{array}{l}132.85 \pm \\
6.12(3)\end{array}$ & $\begin{array}{c}137.91 \pm 6 \\
.27(2)\end{array}$ & $\begin{array}{c}150.34 \\
\text { (1) }\end{array}$ & $\begin{array}{c}154.45 \\
\text { (1) }\end{array}$ & $\begin{array}{c}157.65 \\
\text { (1) }\end{array}$ & $\begin{array}{c}161.23 \\
(1)\end{array}$ & $\begin{array}{c}164.79 \\
(1)\end{array}$ \\
\hline \multicolumn{12}{|c|}{ Sex within the system of rearing } \\
\hline & NS & NS & NS & NS & NS & NS & - & - & - & - & - \\
\hline $\begin{array}{l}\text { Sucklin } \\
\text { g Males }\end{array}$ & $\begin{array}{c}129.91 \pm 6 \\
.37(3)\end{array}$ & $\begin{array}{l}135.66 \pm \\
5.49(3)\end{array}$ & $\begin{array}{l}140.33 \pm \\
5.25(3)\end{array}$ & $\begin{array}{l}144.33 \pm \\
4.57(3)\end{array}$ & $\begin{array}{l}147.42 \pm \\
5.00(3)\end{array}$ & $\begin{array}{r}151.40 \\
+5.12(3)\end{array}$ & $\begin{array}{l}155.45 \pm \\
4.81(3)\end{array}$ & $\begin{array}{l}160.03 \pm \\
4.81(3)\end{array}$ & $\begin{array}{l}163.00 \pm \\
4.67(3)\end{array}$ & $\begin{array}{l}167.61 \pm \\
5.46(3)\end{array}$ & $\begin{array}{l}172.18 \pm \\
5.46(3)\end{array}$ \\
\hline $\begin{array}{l}\text { Sucklin } \\
\text { g } \\
\text { Females } \\
\end{array}$ & $\begin{array}{c}133.46 \pm 7 \\
.80(2)\end{array}$ & $\begin{array}{l}138.49 \pm \\
6.72(2)\end{array}$ & $\begin{array}{l}142.63 \pm \\
6.43(2)\end{array}$ & $\begin{array}{c}138.23 \\
\text { (1) }\end{array}$ & $\begin{array}{c}141.34 \\
\text { (1) }\end{array}$ & $\begin{array}{c}145.78 \\
\text { (1) }\end{array}$ & $\begin{array}{c}150.34 \\
\text { (1) }\end{array}$ & $\begin{array}{c}154.45 \\
\text { (1) }\end{array}$ & $\begin{array}{c}157.65 \\
(1)\end{array}$ & $\begin{array}{c}161.23 \\
\text { (1) }\end{array}$ & $\begin{array}{c}164.79 \\
(1)\end{array}$ \\
\hline $\begin{array}{c}\text { Weaned } \\
\text { Males } \\
\end{array}$ & $\begin{array}{c}95.99 \pm 7 \\
80(2)\end{array}$ & $\begin{array}{l}90.45 \pm 9 \\
.51(1)\end{array}$ & $\begin{array}{c}94.66 \pm 9 \\
.10(1)\end{array}$ & $\begin{array}{c}100.23 \\
(1)\end{array}$ & $\begin{array}{c}104.56 \\
\text { (1) }\end{array}$ & $\begin{array}{c}110.45 \\
(1)\end{array}$ & $\begin{array}{c}114.32 \\
\text { (1) }\end{array}$ & $\begin{array}{c}119.11 \\
(1)\end{array}$ & $\begin{array}{c}123.49 \\
(1)\end{array}$ & - & - \\
\hline $\begin{array}{l}\text { Weaned } \\
\text { Females }\end{array}$ & $\begin{array}{c}106.33 \pm 7 \\
.80(2)\end{array}$ & $\begin{array}{l}110.84 \pm \\
6.72(2)\end{array}$ & $\begin{array}{l}113.89 \pm \\
6.43(2)\end{array}$ & $\begin{array}{l}116.85 \pm \\
5.60(2)\end{array}$ & $\begin{array}{c}124.37 \\
(1)\end{array}$ & $\begin{array}{c}130.05 \\
(1)\end{array}$ & - & - & - & - & - \\
\hline
\end{tabular}

*: Significant at $\mathrm{P} \leq 0.05$;**: Significant at $\mathrm{P} \leq 0.01 ; \mathrm{NS}$ : Non significant 
Table.2 Feeding schedule for weaned calves

\begin{tabular}{|c|c|c|c|c|}
\hline Age of calf & Colostrum & Whole milk & Concentrate & Green fodder \\
\hline 0-3 days & $1 / 10^{\text {th }}$ of Body weight & Nil & Nil & Nil \\
\hline 4-56 days & Nil & $1 / 10^{\text {th }}$ of Body weight & ad lib from $15^{\text {th }}$ day onwards \\
\hline 57-63 days & Nil & $1 / 20^{\text {th }}$ of Body weight & \\
\hline 64-71 days & Nil & $1 / 40^{\text {th }}$ of Body weight & \\
\hline
\end{tabular}

\section{System of rearing wise LBW's in Murrah buffalo calves}

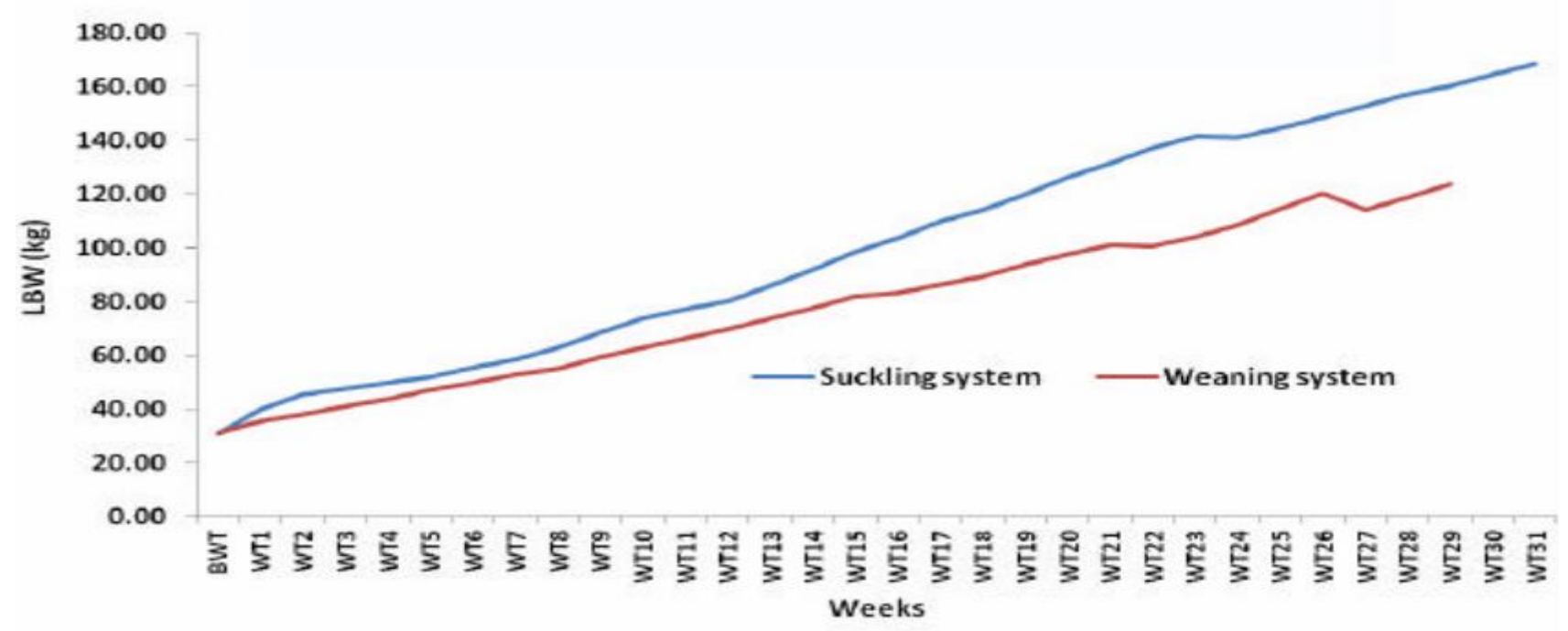

Fig. 1. Effect of rearing system on weekly live body weights of Murrah buffalo calves

Sex-wise weekly live body weights in Murrah buffalo calves

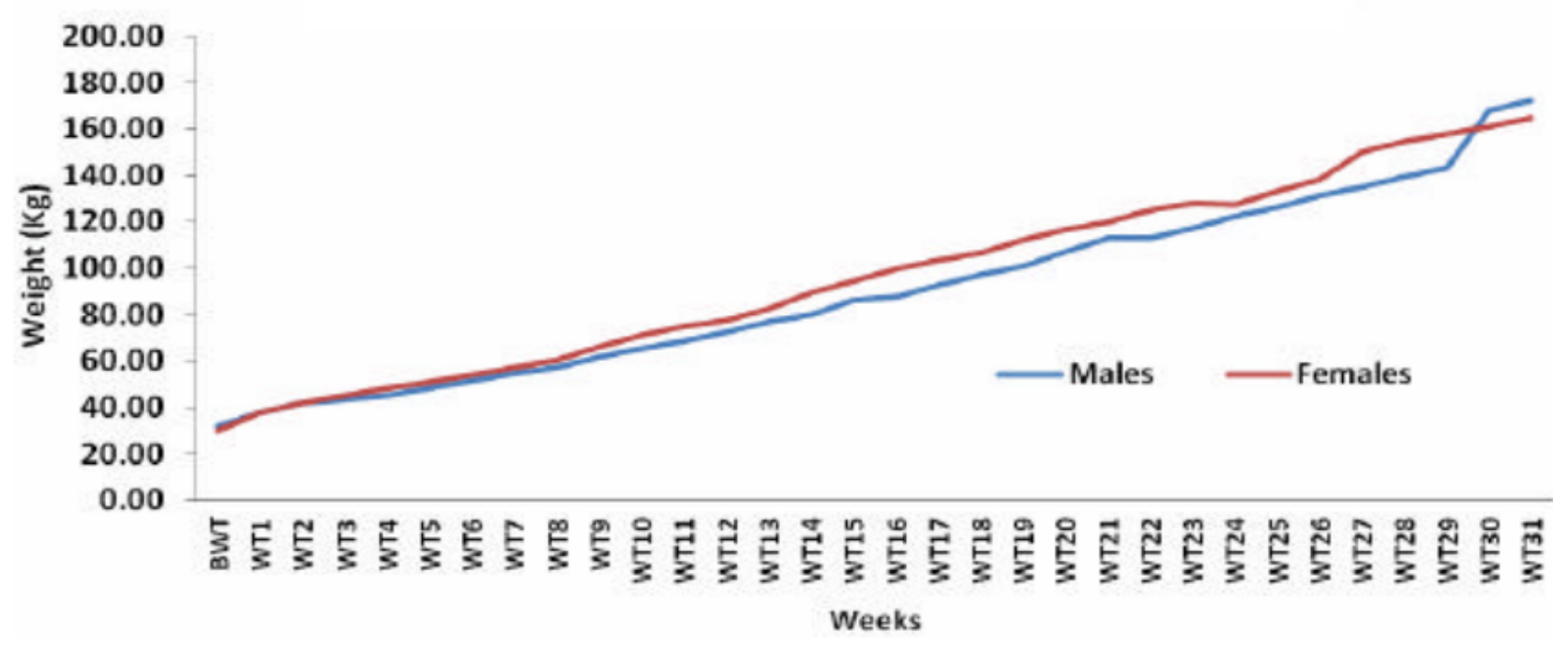

Fig. 2. Effect of sex on weekly livebody weights of Murrah buffalo calves 
Bar-peled et al., (1997) studied the production parameters and weight gain of HF calves that were allowed to suckle from birth to six weeks of age. The weight gain from birth to conception was higher for suckling calves compared to artificially reared calves. During the first 6 weeks, the suckling calves had significantly higher average weight gains over that of control calves (Bar-peled et al., 1997). Krohn (2001) stated that a high daily gain obtained through high milk intake is not necessarily beneficial, because it resulted in a decreased intake of roughage and hence delayed rumen development and increased the difficulties associated with weaning. It was concluded that social interaction between cow and calf in the colostrum period had stimulated the growth of the calf as reflected by daily weight gains of calves (Krohn, 2001). To avoid malnutrition or even death, the farmer has to ensure that all calves are allowed to suckle daily (Krohn, 2001). Suckling calves had a higher weight gain during the suckling period compared to bucket-fed calves. This weight gain was due to higher feed intake in quantity as well as quality of milk. Milk from the dams also includes growth factors such as IGF-I, IGF-II and IGF-binding proteins (mammary-derived growth inhibitor). After the suckling period the calves showed a decreased growth suggesting a greater stress as a result of weaning and difficulties adjusting to solid feed. The HF heifer calves allowed to suckle milk from birth to six weeks of age had higher average daily gains, an earlier age at calving and a tendency for greater milk production than did calves fed milk replacer (Control calves, Bar-peled et al., 1997).

\section{Effect of sex on weekly live body weights in Murrah buffalo calves}

The effect of sex on weekly live body weights was non-significant (Table 1). The LBW's were almost equal for both the sexes at WT1 and WT2, whereas at later stages female calves gained more weight over their male counterparts though, the differences were statistically non-significant (Table 1 and Fig. 2).

\section{Effect of sex (within the rearing system) on weekly live body weights in Murrah buffalo calves}

The effect of sex (within the rearing system) on almost all the LBW's of Murrah buffalo calves from birth to WT31 were nonsignificant (Table 1). Though, the differences were statistically non-significant, males of suckling and weaned system of rearing had more LBW's compared to females in the weaned system.

At the end of the experiment, suckling males showed better performance followed by females of suckling and weaned system of rearing (Table 2 and Fig.2). In contradiction to the present findings, Khoury et al., (1967) reported that early weaned buffalo calves, especially female buffalo calves, showed lower gains at weaning and at subsequent ages up to six months of age as compared to late weaned ones. However, all calves reached at nearly similar weight at 12 months of age. This may be due the fact that male calves were heavier because of which their requirements were higher and finally they consumed greater quantity of milk. It is also in agreement with the report of Mojca Simcic et al., (2007) wherein they reported that males had higher actual and adjusted body weights than females. Krupa et al., (2005) conducted an experiment with actual weaning and yearling weights of six beef breeds of cattle and reported that the sex affect was significant $((\mathrm{P} \leq 0.01)$ on actual weaning and yearling weight. The LSM's were higher for body weights of males compared to that of females for actual and adjusted body weights, too. Actual body weights on the 90 and 365 
days were lower than adjusted body weights of males and females. On the other hand, actual 205-day weight was higher than adjusted body weights for males and females (Mojca Simcic and Cepon, 2007).

Present comparative research work is conducted on Murrah buffalo calves is the first to report their several growth related parameters like weekly body weight gain. More over the results of the current study concluded that suckling calves expressed better live body weights and performed well in earlier stages of their growth than their weaned counterpart but performance was similar in both the groups at later stages of growth. However the present work concluded that weaning can be advised in Murrah buffaloes with scientific and hygienic rearing practices in large farms which is highly economical and these healthy weaned calves will become fruitful livestock assets for the betterment of the livestock sector and in turn contributing major impact to the economy and GDP of growing country like India.

\section{Acknowledgements}

Authors are highly thankful to the Director IVRI Izatnagar for providing necessary facilities to conduct present research work successfully. At the same time the cooperation and help rendered by all the teaching and non-teaching staffs of Section of Livestock Production and Management is thankfully acknowledged.

\section{References}

Arrayet, J. L., Oberbauer, A. M., Famula, T. R., Garnett, I., Oltjen, J. W., Imhoof, J., KehrliJr., M. E. and Graham, T. W. (2002). Growth of Holstein calves from birth to 90 days: The influence of dietary zinc and BLAD status. J. Anim. Sci., 2002, 80:545-552.
Bar-peled U., Robinzon B., Maltz E., Tagari, H., Folman, Y., Bruckental, I., Voet, H., Gacitua, Bell, D.J., Spitzer, J.C. and Bums, G.L. (1998). Comparative effects of early weaning or once-daily suckling on occurrence of postpartum estrus in primiparous beef cows. Theriogenology, 50: 707-715.

Blood, D. C., Radostits, O. M., Gay, C. C., Arundel, J. H., Ikede, B. O. and Mekenzie, R. A. B. C. (1994). Vet. Med. Eighth Ed. ELBS, London.

FAO (2009). Food Outlook: Global Market Analysis. December 2009. pp. 42-51.

FAO (2010). Production Year Book.

Fluharty, F.L., Loerch, S.C., Turner, T.B., Moeller, S.J. and Lowe, G.D. (2000). Effects of weaning age and diet on growth and carcass characteristics in steers. J. Anim.Sci., 78: 1759-1767.

Garnsworthy, P.C. (2005). In: Calf and Heifer Rearing-Principles of Rearing the Modern Dairy Heifer From Calf to Calving. 1st Edi. Nottingham University Press, Nottingham, England.

Green, W. W. and Buric, J. (1953). Comparative performance of beef calves weaned at 90 and 180 day of age. J. Anim. Sci., 12: 561-572.

Khan, A. and Khan, M. Z. (1991). Etiopathology of neonatal calf mortality. J. of Islamic Academy Sci., 4: 159-165.

Khan, A. and Khan, M. Z. (1995). Epidemiological aspects of neonatal calf mortality in the Nili-Ravi buffaloes. Pakistan Vet. J., 15(4): 163-16.

Khan, Zaib Ullah, Khan, Sarzamin, Ahmad Nazir and Raziq, Abdur (2007). J. Agri. and Bio.Sci., 2:22.

Krupa, E., Oracova, M., Polak, P., Huba, J. and Krupova, Z. (2005): Factors affecting growth traits of beef cattle breeds raised in Slovakia. Czech $J$. Anim. Sci., 50: 1, p. $14-21$. 
Mojca, S. and Cepon, M. (2007). Factors affecting actual and adjusted 90-day, 205-day and 365-day weight of Charolais calves. Agriculture, 3(1): 111114.

Pachauri, V.C. and Negi, S.S. (1976). Growth rate of Jersey cross-bred calves of milk and partial milk replacement feeding schedules. Ind. J. Anim. Sci., 46: 383388.

Ranjhan, S.K., Katiyar, R.C., Bhat, P.N. and Raina, B.L. (1972). Studies on growth response of crossbred calves. 1. Effect of limited milk feeding on the growth of crossbred calves up to 3 months of age. Ind. J. Anim. Sci., 42: 754-759.

Saika, T.H, Patel, J.P., Parnerkar, S., Patel, K.S. and Dave, A.D. (1988). Feed efficiency and economics or raising early weaned Kankrej x Jersey and Kankrej x Holstein Friesian calves. Ind. J. Dairy Sci., 41: 162-166.
Sikka, P., Sethi, R.K. and Tomer, A.K.S. (2001). Biochemical studies of suckling vs non suckling buffalo calves in relation to performance of dams. Indian J. Anim. Sci., 72(6): 492-495.

Veissier, I. and Le Neindre, P. (1989). Weaning in calves: its effects on social organization. Appl. Anim. Behav. Sci., 24: 43-54.

Wertz, A. E., Berger, L. L., Walker, P. M., Faulkner, D. B., McKeith, F. K. and Rodriguez-Zas, S. L. (2002). Early weaning and post weaning nutritional management affect feedlot performance, carcass merit, and the relationship of 12th rib fat, marbling score, and feed efficiency among Angus and Wagyu heifers. J. Anim. Sci., 80:28-37.

Wodden, K.G., Speicher, J.A. and Huber, J.A. (1968). Effect of feeding system on feed and labour cost and rates of gain in dairy calves. J. Dairy Sci., 51: 971.

\section{How to cite this article:}

Kantharaja, K.J., A.K.S. Tomar, O.R. Nataraju and Naveen Kumar, B.T. 2018. Early Growth Performance Comparison of Weaned and Suckling Murrah Buffalo Calves under Institutional Situations. Int.J.Curr.Microbiol.App.Sci. 7(05): 723-733. doi: https://doi.org/10.20546/ijcmas.2018.705.087 